\title{
Block-based Adaptive Image Watermarking Scheme using Visual Perception Model in DCT Domain
}

\author{
F. Namazi \\ Faculty of Electrical and Computer \\ Engineering \\ Bobol University of Technology
}

\author{
M. R. Karami \\ Faculty of Electrical and Computer \\ Engineering \\ Bobol University of Technology
}

\author{
S. B. Ramazannia \\ Faculty of Electrical and Computer \\ Engineering \\ Mazandaran Institute of \\ Technology
}

\begin{abstract}
In this paper, an improved DCT-based watermarking technique has been presented, which exploits human visual model for adapting the watermark data to local properties of the host image. In order to achieve more robustness and transparency, watermark has been embedded into the middle frequency components of the image. These components are significant in high textural regions so that the visibility of the image would not be affected and the watermark will not be removed by compression. Experimental results are provided to demonstrate that embedded watermarks can be almost fully extracted from the JPEG-compressed images with very high compression ratios and other kind of attacked images.
\end{abstract}

Keywords:Digital Watermarking, Discrete cosine transformation, Human Visual System

\section{INTRODUCTION}

During the past two decades, the rapid development of the Internet has introduced a new set of challenging security problems. One of the most important problems is how to discourage the unauthorized duplication of digital data. Digital watermarking has been proposed as a way to resolve this problem. Digital watermarking is a technique to embed additional information into an image, audio, video and text, which can be later extracted or detected for variety of purposes including copyright protection, copy control, broadcast monitoring and image authentication purposes [1].The conventional watermarking techniques can be categorized into two classes: spatial domain and transform domain methods. Spatial domain techniques embed the watermark by directly modifying the intensity, color, or values of the pixels [2]. These techniques generally require a lower computational cost than transform domain techniques, hence are generally easier to implement [3]. Transform domain techniques require filtering the image into frequency channels. Then the watermark is inserted into one or more channels based on certain criteria. Recently many robust watermarking schemes using the transform domain approach have been proposed. The important requirements of such watermarks are imperceptibility and robustness. Watermark imperceptibility means that the watermark should be hidden in the cover image in such a way that the changes of the cover images are visually imperceptible. Robustness of a watermark is the ability to extract the watermark correctly even if intentional or unintentional attacks are applied on the watermarked image. In terms of embedding strength against different attacks, it should be as high as possible. Since watermark directly affects the original image, so the higher embedding strength leads to the lower quality of the watermarked image. Therefore, the tradeoff between the robustness and the imperceptibility is inevitable [4]. A balance is achieved by exploiting the characteristics of the Human Visual System (HVS). In this way, the strength of the watermark is adapted to the features of the original image to guarantee the maximum possible imperceptibility of the watermarked image [5, 6].

Many watermarking techniques based on DCT have been presented [7-8]. Since JPEG/MPEG coding is based on a DCT decomposition, embedding a watermark in the DCT domain makes it possible to integrate watermarking with image/video coding. In [9] designed a class of perceptual watermarks that exploit perceptual information in the watermarking process known as perceptual watermarks. These watermarking algorithms depend not only on the frequency response of the human eye, but also the properties of the image itself, to decide the watermarking strength. In [10] suggested a technique to embed multiple watermarks in the DCT domain using different keys and a technique called iterative watermark embedding, which embeds watermarks into JPEG compressed images. In [11] proposed a chaos-based DCT-domain watermarking algorithm. The cat map is used to shuffle the watermark bits, which ensures the watermark bits spread in all the regions of the host image. The logistic map is employed to determine which blocks of the DCT coefficients are selected for watermark embedding. Mapping table and host image are not needed for watermark extraction. The ones who don't know the keys can't extract the watermark from the watermarked image. In this paper, we propose an adaptive DCT-based watermarking technique considering HVS characteristics, where the watermarking is performed by using a block-based approach. For a block-based watermarking scheme, a high textural block is favored for embedding watermark bits to achieve high imperceptibility. Therefore, the localizations of the watermark bits are adjusted adaptively in accordance with the human visual system and image characteristics, which makes the applied changes perceptually invisible. The DCT is then applied to the selected image blocks subsequently. The watermark is embedded into the selected blocks by modifying the middle frequency components of each block for preserving the visual quality of watermarked images and increasing the robustness of watermark. The technique can satisfy both imperceptibility and robustness requirements of the watermarking system. This paper is organized as follows. The background on HVS is explained in section 2 . The proposed block-based adaptive image watermarking scheme using visual perception model in DCT domain is described in Section 3. In Section 4, the performance of the proposed watermarking scheme in the form of transparency and robustness is evaluated and the comparison 
with the existing watermarking scheme is made. The conclusions are finally described in Section 5.

\section{LITERATURE REVIEW}

Based on the knowledge of the structure of the human eye and human visual system, some characteristic of human eyes have been found as follows [6]:

(1) Disturbances are much less visible in highly textural regions than in the uniform areas.

(2) Contours are more sensitive to noise addition than highly textured regions but less than flat areas.

(3) Disturbances are less visible over dark and bright regions.

Human visual systems (HVS) have been studied by a lot of individuals. Bartolini and Barni [12] followed the three mentioned rules to develop their masking. To choose the frequency components where the watermark was embedded, first of all, the image is passed through a band pass filter. Second, using the edge detection to locate the edge area in the original image. The third and fourth steps applied the median filter with different thresholds to determine the areas with brightness and darkness. And finally, from the summation of previous steps results a binary image is obtained. An offset value was given to the final summation which resulted in the minimum amount of watermark energy inserted. One important advantage of this method is that, it can locate where the watermark should be strong or weak. Some advantages of this algorithm are that determining the offset value for different images are quite difficult, moreover it does not give the mask value of the image but only the locations of the watermark. The algorithm was developed by Qi [13]. He determined the luminance masking, texture masking and edge masking based on the image features, and then combine these three masking together to get a comprehensive final masking for the watermark in the spatial domain as shown in Figure 2.(a)

In [14], Watson proposed a new masking effect called entropy masking, which is due to unfamiliarity of the observer. In fact, entropy masking is due to weakness of human brain in processing simultaneous complex phenomena. By performing a number of experiments, Watson showed that this masking phenomenon is distinct from previously known masking effects such as contrast masking.Entropy gives a measure of complexity of the image. Complex textures tend to have higher entropy. (Shown Fig.2.(b))

Another stochastic model of the host image is applied to an adaptive watermark by computing noise visibility function (NVF) with non-stationary Gaussian model. In this case, NVF can be expressed by

$$
N V F(i, j)=\frac{1}{1+\sigma_{x}^{2}(i, j)}
$$

Where $\sigma_{\mathrm{x}}^{2}(\mathrm{i}, \mathrm{j})$ denotes the local variance of the host image in a window centered on the pixel with coordinates $(i, j)$. NVF is calculated from original image during embedding process, as showed in the work of Voloshynovskiy [15]. By applying NVF, the watermark in texture and edges becomes stronger than in flat areas and the proposed embedding algorithm is adaptive. In the regions with edges and texture, NVF has a value near 0 while in smooth regions; the value of NVF is near 1 . However, it could not deal with flat areas.(Shown Fig.2.(c))

\section{PROPOSED SCHEME}

The block-based DCT transform segments to image nonoverlapping blocks and applies DCT to each block. These results in three frequency sub-bands: low frequency sub-band, mid-frequency sub-band and high frequency sub-band. Fig.1 shows different frequency bands in one $8 * 8$ block.

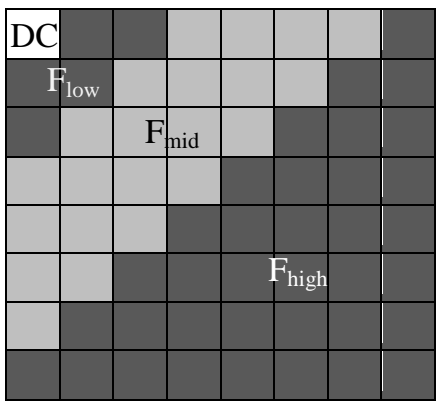

Fig.1. Different frequency bands in one $8 * 8$ block

Experimental results show that embedding in the low frequency components of the block, which carry important perceptual information and are dominant components of uniform regions, will cause obvious quality distortion in the watermarked image. Middle frequency components are significant in high textural regions so that by watermark embedding in them the visibility of the image will not be affected and the watermark will not be removed by compression. High frequency components show detail information of the image that are usually removed through compression and noise attacks.

After reviewing different masking models, we understand that high texture regions play important role in human visual system. As mentioned above, the middle frequencies of the DCT represent texture of the blocks. In other words, DCT blocks with the most energetic middle frequency coefficient are located in high texture regions. The selected blocks in the proposed method are very similar to the other methods. (Shown in Figure 2(d))

As will be explained later, in embedding process the middle frequency components energy must be calculated. Therefore, no additional calculations to create the mask are needed.

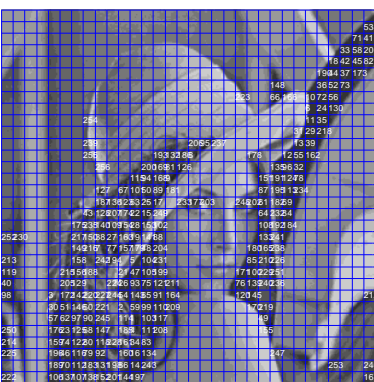

(a)

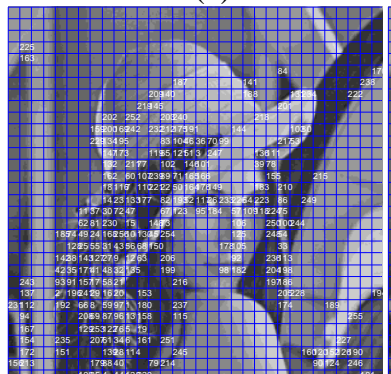

(c)

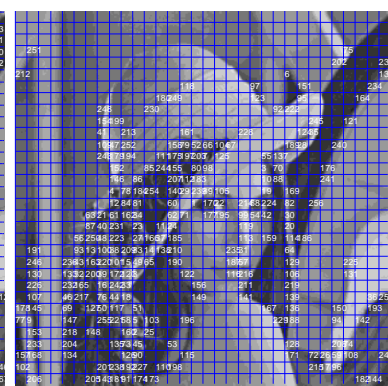

(b)

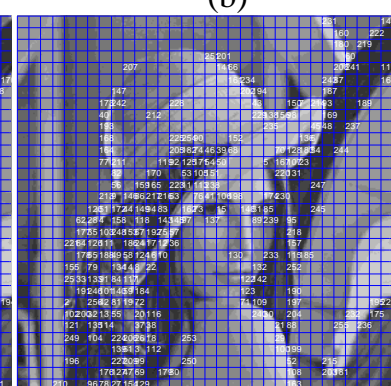

(d)
Fig.2. compression of selected blocks (a)HVS masking[16], (b)Entropy masking[17], (c)NVF masking[18], (d) Proposed masking 


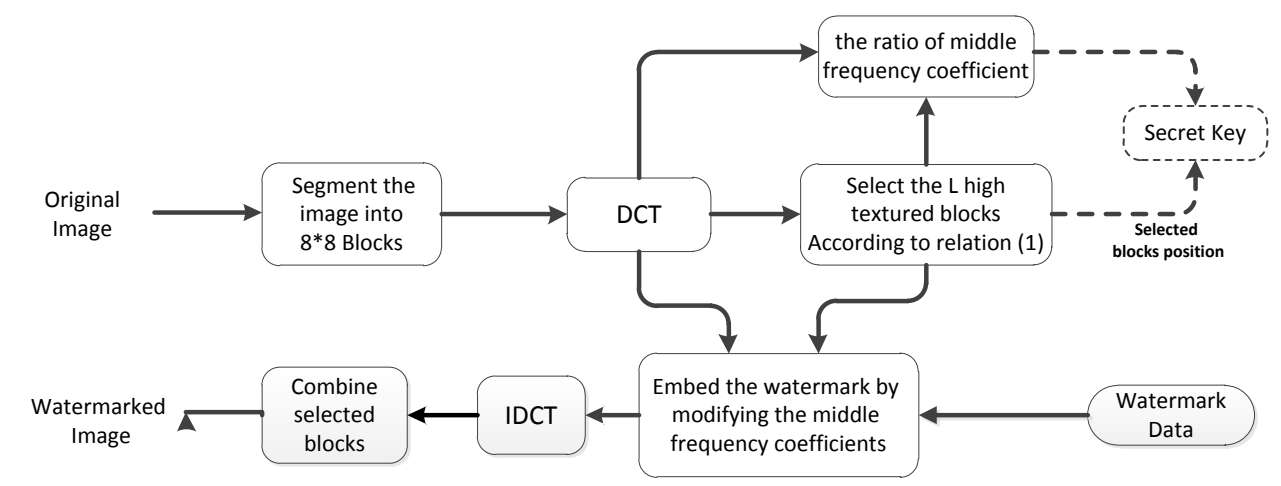

Fig .3. The procedure of watermark embedding

\subsection{Watermark Embedding Scheme}

In this paper, we embed the binary watermark in DCT domain. The overall block diagram of the proposed method is presented in Fig. 3. The watermark embedding procedure can be summarized in the following steps.

1. Theoriginal image is divided into $\mathrm{N} \times \mathrm{N}$ nonoverlapping blocks.

2. The DCT is then applied to each of the blocks.

3. In order to embed the watermark data into the more textured regions, L blocks with (the most energetic middle frequency coefficients) hightexture are selected. This high-texture thresholding scheme is carried out so that the invisibility of the watermark insertion is assured.

$$
\text { index }_{l}=\arg \max \left(D_{5}+D_{6}\right) \quad, \quad 1 \leq l \leq L
$$

Where

$$
\begin{aligned}
& D_{5 i}=\sum_{k=0}^{5}\left|D C T_{i}(k, 6-k)\right| \\
& D_{6 i}=\sum_{k=0}^{6}\left|D C T_{i}(k, 7-k)\right| \quad, \quad 1 \leq i \leq \frac{M \times N}{8 \times 8}
\end{aligned}
$$

Where $\mathrm{M}$ and $\mathrm{N}$ are the image size.

Selected blocks in proposed method have been shown in Fig.2-d.

4. Before inserting the watermark in the selected blocks, the ratio of middle frequency coefficients for each block $\left(d_{1}\right)$ is calculated as (5)and stored as a key.

$$
d_{l}=\frac{D_{5 l}}{D_{6 l}} \quad, \quad 1 \leq l \leq L
$$

5. In this step, the watermark data $M=\left\{m_{1}, m_{2} \ldots\right.$ $\left.\mathrm{m}_{\mathrm{L}}\right\}$ is embedded by modifying the amplitude of the selected coefficients in the following way:

$$
\begin{aligned}
& \text { if } m_{l}=1,\left\{\begin{array}{l}
D_{w 5 l}=\alpha \times D_{5 l} \\
D_{w 6 l}=\frac{1}{\alpha} \times D_{6 l}
\end{array}\right. \\
& \text { if } m_{l}=0,\left\{\begin{array}{l}
D_{w 5 l}=\frac{1}{\alpha} \times D_{5 l} \\
D_{w 6 l}=\alpha \times D_{6 l}
\end{array}\right.
\end{aligned}
$$

Where $\mathrm{D}_{51}$ and $\mathrm{D}_{61}$ are the DCT coefficients, $\mathrm{D}_{\mathrm{W} 51}$ and $\mathrm{D}_{\mathrm{W} 61}$ are the watermarked DCT coefficients of L selected blocks, and $\alpha$ is the watermark strength factor and has a value larger than one. A larger value for $\alpha$ leads to more robustness and lower transparency and vice versa.
6. In the final step, the inverse DCT is applied to all selected blocks subsequently and the watermarked image is constructed.

\subsection{Watermark extracting Sechme}

The steps of the proposed watermark extracting algorithm are very similar to that of watermark embedding algorithm, except for the step of watermark information extraction. The procedure for watermark extraction is quite simple and it doesn't need any assistance of the original host image. Note that two key parameters are needed in the watermark extraction procedure: the record of selected image blocks that were embedded with watermark bits and the ratio of middle frequency coefficient $d_{1}$ . The watermark extraction procedure can be described in four steps as follows. The block diagram of the proposed watermark extracting method is shown in Fig. 4.

1. The watermarked image is divided into $\mathrm{N} \times \mathrm{N}$ non overlapping blocks.

2. The DCT is applied to the blocks where the watermark data have been embedded during the watermark embedding process.

3. The coefficients representing the most energetic direction in each block are selected.

$$
d_{w l}=\frac{d_{w 5 l}}{d_{w 6 l}} \quad, \quad 1 \leq l<L
$$

4. The watermark data is extracted from the coefficients obtained in step 3, using (9) and (10).

$$
\begin{aligned}
& \text { if } \frac{d_{w l}}{d_{l}}>1, \text { Then } \hat{m}_{l}=1 \\
& \text { if } \quad \frac{d_{w l}}{d_{l}}<1 \text {, Then } \hat{m}_{l}=0
\end{aligned}
$$

Where $\widehat{m}_{l}$ is the extracted watermark data, $d_{l}$ and $d_{w l}$ are the ratio of original image DCT coefficients and the ratio of watermarked DCT coefficients respectively.

As it is known, there is a tradeoff between the imperceptibility and robustness of a digital watermarking system. The proposed system to control this tradeoff, an embedding coefficient $(\alpha)$ is considered. From equation 5 and 6 inembedding process, the following relationships can beinferred.

$$
\text { if } \quad \hat{m}_{l}=1 \Rightarrow \frac{d_{w l}}{d_{l}}=\alpha^{2}
$$




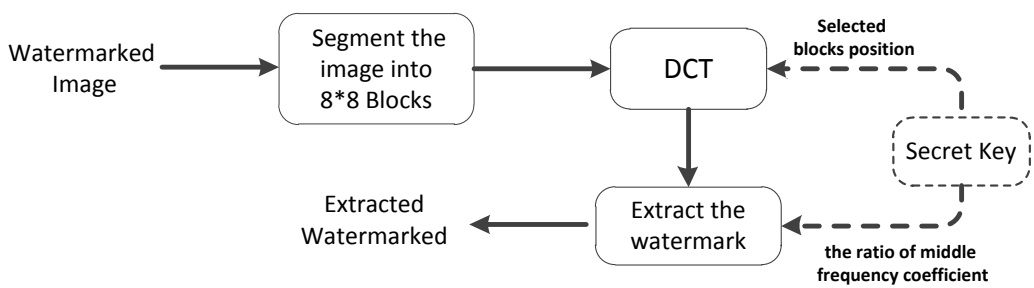

Fig .4. The procedure of watermark extracting

$$
\text { if } \quad \hat{m}_{l}=0 \Rightarrow \frac{d_{w l}}{d_{l}}=\frac{1}{\alpha^{2}}
$$

Since $\alpha$ is greater than 1. It is obvious that for higher embedding coefficients, variations in DCT coefficients are higher and in turn, the quality of the watermarked image is lower. On the other hand, since the strength of the embedded watermark is higher, the robustness of the watermark will increase. In this way, the strength of the watermark is adapted to the feature of the original image by selecting high texture blocks to guarantee the maximum possible imperceptibility and robustness of the watermark.

\section{EXPERIMENTALRESULTS}

Inorder to measure the actual performance of the proposed scheme, we conducted experiments with different well-known $256 \times 256$ grayscale test images. In all experiments, $\mathrm{N}$ was set to 8 and therefore, 904 blocks were obtained for each image.The number of selected blocks, L was 64. However, different number of blocks can be selected for watermark embedding to maximum value of 904 for a $256 \times 256$ image. This is the maximum capacity of our watermarking scheme. Increasing L will result in decreasing the performance of the scheme against attacks. The watermark signal, containing 0 bits and 1 bit, was generated randomly. Also, the value of $\alpha$ was chosen in a way that the peak signal to- noise ratio (PSNR) for all watermarked images were about $45 \mathrm{~dB}$. This PSNR was selected in order to assure invisibility of the watermark insertion and also achieving high robustness to different attacks. The original and watermarked images are shown in Figs. 5 (a) and (b). Ascan be seen there is no perceptual difference between the original and watermarked images.

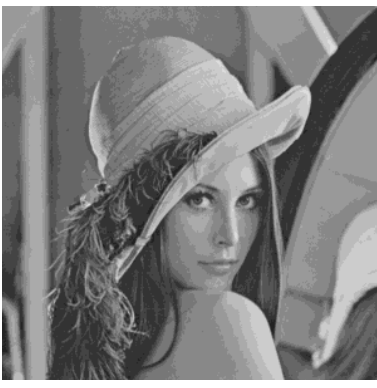

(a)

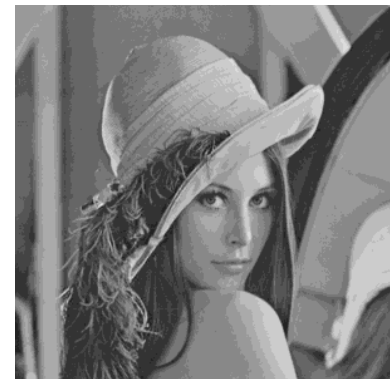

(b)
Fig.5: (a) Original Test Images \&(b) Watermarked Test Image

\subsection{Addition of noise}

Addition of noise is a method to estimate the robustness of the watermark. Generally, addition of noise is responsible for the degradation and distortion of the image. The watermark information is also degraded by noise addition and results in difficulty in watermark extraction. Salt and pepper noise can be roughly considered as a signal with lots of high frequency. Hence, our watermark embedding and detecting scheme should be robust against the salt and pepper noise because the watermark is embedded in middle frequencies of the image. The reader may refer to Fig. 6 for detailed performance of our watermarking scheme against the salt and pepper noise attack.

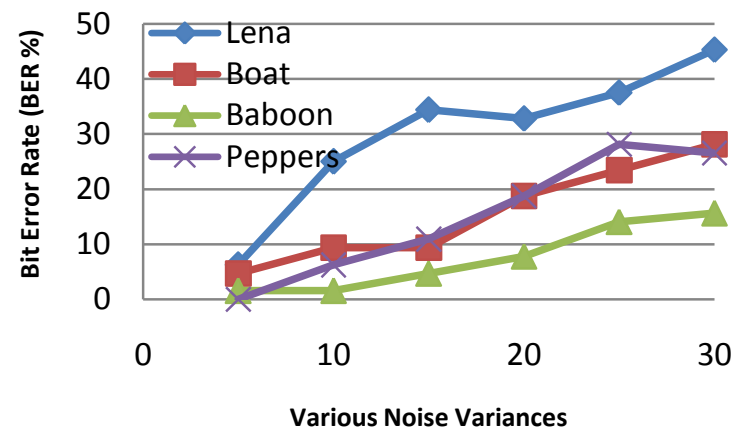

Fig. 6. BER of watermark extraction after salt and peppers noise attack for various variances.

\subsection{JPEG compression}

One of the most important attacks, which every image watermarking system should be robust to, is the JPEG compression attack. The robustness of the scheme against JPEG lossy compression is investigated by compressing the watermarked signal using JPEG compressor with different impact factors and then trying to extract the watermark signal. The BER of watermark extraction and PSNR of the attacked image are presented in Table I. As can be seen, the proposed method extracts the watermarksignal without error for all images even after JPEG compression with quality factor equal to $50 \%$.

Table1. Robustness of the proposed scheme against JPEG compression with different quality factors

\begin{tabular}{clcccc}
\hline $\begin{array}{c}\text { Quality } \\
\text { Factors }\end{array}$ & & Lena & Boat & Baboon & Peppers \\
\hline \multirow{2}{*}{$10 \%$} & PSNR & 35.4 & 33.25 & 30.41 & 34.33 \\
& BER(\%) & 10.93 & 3.12 & 0 & 3.13 \\
\hline \multirow{2}{*}{$20 \%$} & PSNR & 37.71 & 34.63 & 31.2 & 35.98 \\
& BER(\%) & 3.12 & 4.68 & 0 & 1.56 \\
\hline \multirow{2}{*}{$30 \%$} & PSNR & 39.17 & 35.55 & 31.76 & 36.93 \\
& BER(\%) & 0 & 0 & 0 & 0 \\
\hline \multirow{2}{*}{$40 \%$} & PSNR & 40.07 & 36.18 & 32.24 & 37.47 \\
& BER(\%) & 0 & 0 & 0 & 0 \\
\hline \multirow{2}{*}{$50 \%$} & PSNR & 40.82 & 36.8 & 32.68 & 37.94 \\
& BER(\%) & 0 & 0 & 0 & 0 \\
\hline
\end{tabular}

In order to evaluate the robustness of the proposed method in comparison to other schemes, we compared our watermarking 
algorithm with method proposed in [11]. In Table 2, the PSNR and BER values are provided, and present a comparison in terms of imperceptibility and robustness. In this comparison we used the Lena as host image. As can be seen, our methodhas superior imperceptibilityand robustness in comparison with Yantao's method.

Table2.Scheme Compression in terms of imperceptibility and robustness of the proposed scheme with Yantao's scheme

\begin{tabular}{|c|c|c|}
\hline \multirow{2}{*}{ attack } & Yantao in [11] & Proposed method \\
\hline & $\operatorname{BER}(\%)$ & $\operatorname{BER}(\%)$ \\
\hline JPEG90 & 0 & 0 \\
\hline JPEG70 & 0.27 & 0 \\
\hline JPEG50 & 0 & 0 \\
\hline JPEG40 & 6.23 & 0 \\
\hline JPEG35 & 8.94 & 0 \\
\hline average filtering $(3 \times 3)$ & 29.35 & 0 \\
\hline median filtering $(3 \times 3)$ & 20.75 & 1.56 \\
\hline gaussian filtering $(3 \times 3)$ & 4.93 & 0 \\
\hline gaussian filtering $(5 \times 5)$ & 5.08 & 0 \\
\hline cropping $25 \%$ & 11.96 & 31.25 \\
\hline histogram Equalization & 33.45 & 0 \\
\hline rotate 5 then rerotate- 5 & 11.55 & 0 \\
\hline $\begin{array}{l}\text { Add noise(salt and } \\
\text { pepper }(0,0.01)\end{array}$ & 27.29 & 3.125 \\
\hline
\end{tabular}

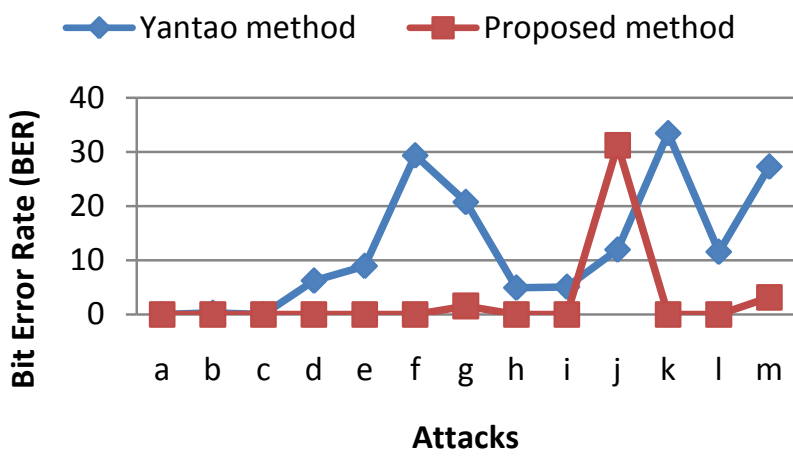

Fig. 7: Compression of the robustnessof the proposed scheme with Yantao'smethod: (a) JPEG Compression Q = 90\%;(b) JPEG Compression Q = 70\%; (c JPEG

Compression $Q=50 \%$ ); (d) JPEG Compression $Q=40 \%$; (e) JPEG Compression $Q=350 \%$; (f) Average filter [3×3]; (g) Median filter [3×3]; ( h) Gaussian filter [3×3]; (i) Gaussian filter [5×5]; (j) cropping 25\%; (k) Histogram equalization; (l) rotation30 degree; (m) Salt \& peppers $1 \%$ Fig.7. depicts the BER comparisons between proposed and Yantao [11] algorithms except of cropping attack.

\section{CONCLUSION}

In this paper, an image watermarking technique based on DCT and human visual characteristics is presented. The technique fully exploits the respective feature of DCT which efficiently represents intrinsic algebraic properties of an image. The use of
HVS characteristics helps to select watermark embedding regions for a good compromise between robustness and quality of the watermarked image. Simulation results of the proposed technique have shown both the significant improvement in imperceptibility and the robustness under different types of image processing operations, especially for the JPEG compression with different quality factors. As for future work, more perceptual features and more advanced perceptual models will be utilized.

\section{REFERENCES}

[1] N. K Kalantari, S. M. Ahadi, F. Marvasti, "A Robust Image Watermarking in the Ridgelet Domain Using Universally Optimum Decoder," IEEE Trans. Circuits and Systems for VideoTechnology. 20 (3) pp.396-406,(2010).

[2] C.-H. Chang, Z. Ye, M. Zhang, Fuzzy-ART based adaptive digital watermarking scheme, IEEE Transactions on Circuits and Systems for Video Technology 15 (1)pp.6581 (2005)

[3] I.J. Cox, J. Kilian, T. Leighton and T. Shamoon, "Secure Spread Spectrum Watermarking for Multimedia", IEEE Trans. on Image Processing, pp. 1673-1687, 1997.

[4] H. Qi, D. Zheng, J. Zhao, "Human visual system based adaptive digital image watermarking,"Signal Process. 88,PP. 174-188, Jul. 2008.

[5] O. T.-C. Chen and W-Ch. Wu,"Highly Robust, Secure, and Perceptual-QualityEcho Hiding Scheme," IEEE Transactionson Audio,speech and language processing, Vol. 16, NO. 3, pp.629-638, 2008.

[6] M. A Akhaee, S. M. E Sahraeian, F. Marvasti, "Contourlet-Based Image Watermarking Using Optimum Detector in a Noisy Environment," IEEE Trans. Image Process. 19 (4) pp.969-980,(2010).

[7] S.D. Lin a, S-C. Shie b, J.Y. Guo, "Improving the robustness of DCT-based image watermarking against JPEG compression," Computer Standards \& Interfaces 32, pp.54-60,2010.

[8] S. Lin, C. Chin,. "A Robust DCT-based Watermarking for Copyright Protection," IEEE Trans. Consumer Electronics, 46(3): pp.415-421, 2000.

[9] R.B. Wolfgang, C.I. Podilchuk and E.J. Delp, "Perceptual Watermarks for Digital Images and Video," Proceedings of the IEEE, Vol. 87, No. 7, pp. 1108-1126, 1999.

[10] Peter H.W. Wong, Oscar C. Au and Y.M. Yeung, "A Novel Blind Multiple Watermarking Technique for Images," IEEE Trans. on Circuits and Systems for VideoTechnology, Vol. 13, No. 8, pp. 813-830, 2003.

[11] Z. Yantao, M. Yunfei, L. Zhiquan, "a robust chaos-based DCT-domain watermarkingalgorithm," 2008 International Conference on Computer Science and Software Engineering, pp.935 - 938, 2008.

[12] F. Bartolini, M. Barni, V. Cappellini, A. Piva, Mask building for perceptually hiding frequency embedded watermarks, in: Proceedings of the IEEE ICIP98 1 (October 1998) 450-454.

[13] H. Qi, D. Zheng, J. Zhao."Human visual system based adaptive digital image watermarking". Signal Process. 88,PP. 174-188, Jul. 2008.

[14] A. B. Watson, R. Borthwick, and M. Taylor, "Image quality and entropy masking," in SPIE Proceedings, San Diego, California, vol. 3016, 1997.

[15] S. Voloshynovskiy, A. Herrigel, N. Baumgaertner, T. Pun, A stochastic approach to content adaptive digital image watermarking, in: Proceedings of the International Workshop on Information Hiding, Lecture Notes in Computer Science, vol. LNCS 1768, 1999, pp. 212-236. 